\title{
New Tumor Event After Initial Treatment Unknown
}

National Cancer Institute

\section{Source}

National Cancer Institute. New Tumor Event After Initial Treatment Unknown. NCI

Thesaurus. Code C160287.

It is unknown if a new tumor exists after the initial treatment. 